\title{
Segurança do paciente em serviços de tomografia computadorizada: uma revisão integrativa
}

Késsya Dantas Diniz ${ }^{1}$, Isabelle Katherinne Fernandes Costa ${ }^{2}$, Richardson Augusto Rosendo da Silva ${ }^{3}$

\author{
${ }^{1}$ Enfermeira, Mestre em Enfermagem. \\ Discente do Programa de Pós-Graduação \\ em Enfermagem, nível Doutorado, da \\ Universidade Federal do Rio Grande do \\ Norte. Natal, RN, Brasil. E-mail: \\ kessyadantas@yahoo.com.br. \\ ${ }^{2}$ Enfermeira, Doutora em Enfermagem. \\ Professora Adjunta da Universidade \\ Federal do Rio Grande do Norte. Natal, RN, \\ Brasil. E-mail: isabellekfc@yahoo.com.br. \\ ${ }^{3}$ Enfermeiro, Doutor em Ciências da \\ Saúde. Professor Adjunto da Universidade \\ Federal do Rio Grande do Norte. Natal, RN, \\ Brasil. E-mail: rirosendo@hotmail.com.
}

Recebido: 30/05/2015.

Aceito: 29/06/2016.

Publicado: 21/12/2016.

Como citar esse artigo:

Diniz KD, Costa IKF, Silva RAR. Segurança do paciente em serviços de tomografia computadorizada: uma revisão integrativa. Rev. Eletr. Enf. [Internet]. 2016 [acesso em: ___];18:e1189. Disponível em: http://dx.doi.org/10.5216/ree.v18.35312.

\section{RESUMO}

Objetivou-se identificar os elementos essenciais para oferecer uma assistência segura aos pacientes submetidos ao exame de tomografia computadorizada. Revisão integrativa da literatura nas bases de dados Medline, SCOPUS, Web of Science e CINAHL, norteada pela questão: quais são os elementos essenciais para oferecer uma assistência segura ao paciente submetido ao exame de tomografia computadorizada? Selecionou-se 23 artigos, dos quais 50\% abordaram a otimização, monitoramento, qualidade e proteção em relação às doses de exposição à radiação; $25 \%$ enfocaram o contraste utilizado na tomografia; e $25 \%$ os cuidados de enfermagem e gestão de casos específicos. As evidências para a assistência segura ao paciente submetido à tomografia computadorizada apontam para a necessidade de, minimizar a realização de exames desnecessários e consequente exposição à radiação, garantir a proteção radiológica, disponibilizar atendimento adequado nas reações adversas, recursos humanos qualificados e indicadores de qualidade de assistência confiáveis.

Descritores: Diagnóstico por Imagem; Segurança do Paciente; Tomografia.

\section{INTRODUÇÃO}

O desenvolvimento da ciência e o crescente emprego das tecnologias duras na área da saúde, com a utilização de aparelhos modernos, complexos e sofisticados que possibilitam exames de imagens de alta definição para o diagnóstico e tratamento de enfermidades, têm permitido o diagnóstico precoce e o tratamento de doenças, contribuindo para a melhora das condições de vida e saúde da população ${ }^{(1-3)}$. 
Esse avanço tecnológico vem sendo acompanhado por mudanças de paradigmas na atenção à saúde, apontando para a necessidade de segurança e redução de riscos ao paciente, estabelecimento e utilização de indicadores de avaliação da qualidade da assistência, implementação de protocolos assistenciais, prevenção e análise de ocorrência de eventos adversos, embasados em evidências científicas ${ }^{(4-6)}$.

A segurança do paciente é um componente crítico da melhoria da qualidade do cuidado da saúde em todo o mundo, visto que constitui globalmente um grave problema de saúde pública. Estimativas de países desenvolvidos indicam que pelo menos um em cada dez pacientes que recebem cuidados assistenciais hospitalares sofrem danos, denominados eventos adversos. As consequências desses eventos podem ser graves ou fatais, e extremamente dispendiosas para suas vítimas e para os sistemas de cuidados de saúde ${ }^{(7-}$ 9).

Por consequência, cresce o reconhecimento de profissionais e gestores da saúde sobre a importância da promoção de uma cultura de segurança que envolva os profissionais de saúde e a organização como um todo. Nesse contexto, a segurança do paciente é um importante indicador da qualidade dos serviços prestados $^{(10-11)}$.

Nos Centros de Diagnósticos por Imagem (CDI), os profissionais devem ser capacitados para atender as necessidades dos pacientes em cada modalidade de exames diagnóstico, como no setor de Tomografia Computadorizada $(\mathrm{TC})^{(3)}$. A realização dos exames com qualidade, minimizando custos e reduzindo a quantidade de radiação ao paciente, ao profissional e ao meio ambiente, requer um esforço organizado com o objetivo de assegurar que as imagens diagnósticas produzidas tenham qualidade para fornecer informações adequadas para o diagnóstico seguro ${ }^{(4)}$.

Dessa forma, ao direcionar o olhar aos serviços de TC, os profissionais possuem um importante papel na prevenção, detecção e tratamento dos eventos adversos que podem ser causados pelo uso de contraste iodado, bem como no fornecimento de informações necessárias para a adequada realização do exame e o consequente diagnóstico seguro ${ }^{(3)}$.

A justificativa para a realização do presente estudo se baseia na carência de pesquisas que reúnam elementos essenciais para oferecer uma assistência segura aos pacientes submetidos à TC, havendo a necessidade de buscar evidências na literatura nacional e internacional sobre o tema, visando qualificar a assistência de enfermagem no intuito de garantir ao paciente a realização desse exame de forma mais segura.

Ademais, a literatura revela lacunas relacionadas à escassez de pesquisas como revisões sistemáticas, meta-análises, ensaios clínicos e ensaios clínicos randomizados bem delineados, que proporcionem níveis de evidências fortes sobre os subsídios essenciais para oferecer uma assistência segura aos pacientes submetidos à tomografia computadorizada, bem como o impacto da qualidade e segurança da assistência prestada a essa clientela.

Além disso, esse estudo é relevante por trazer informações acerca da promoção da segurança de pacientes no $\mathrm{CDI}$, onde se observa o aumento da realização de exames de imagem, devido a crescente evolução dos recursos diagnósticos e pelo seu alto poder de acurácia ${ }^{(5-7,9-11)}$. Assim, se trata de um novo 
espaço para a gestão do risco, gerenciamento do processo de cuidar, investigação e ensino em saúde. Nesse sentido, pesquisas devem ser cada vez mais desenvolvidas, a fim de se produzir conhecimentos que promovam a sustentabilidade de uma cultura de segurança positiva no âmbito das organizações de saúde ${ }^{(10-}$ 13)

Desse modo, este estudo teve como objetivo identificar os elementos essenciais para oferecer uma assistência segura aos pacientes submetidos ao exame de tomografia computadorizada, a partir de evidências da literatura.

\section{MÉTODOS}

Revisão integrativa da literatura, a qual permite a busca, a avaliação crítica e a síntese de múltiplos estudos publicados, possibilitando conclusões relacionadas a uma determinada área de estudo ${ }^{(14)}$.

Para a operacionalização desse estudo foram percorridas as seguintes etapas: elaboração da questão de pesquisa, definição do objetivo, determinação dos critérios de inclusão e exclusão das publicações, definição das informações a serem coletadas dos estudos selecionados, análise, interpretação e síntese dos resultados da revisão ${ }^{(14)}$.

Como forma de direcionar esta revisão, foi utilizada a seguinte questão norteadora: Quais são os elementos essenciais para se oferecer uma assistência segura aos pacientes submetidos ao exame de tomografia computadorizada?

O levantamento das publicações ocorreu no período de outubro e novembro de 2015 nas referidas bases de dados: Medical Literature Analysis and Retrieval System Online (Medline), SCOPUS, Web of Sciense, Cumulative Index to Nursing Allied Health Literature (CINAHL), não sendo estabelecidos limites quanto ao ano de publicação.

A busca foi realizada em pares, e na ordem em que essas bases de dados aparecem descritas, de forma que as publicações que se encontravam indexadas em mais de uma, foram selecionadas na primeira busca.

Para o refinamento dos artigos estabeleceram-se como critérios de inclusão: artigos que abordassem a segurança do paciente na realização de exames por imagem, mais especificamente tomografia computadorizada; disponíveis na íntegra, nas bases de dados supracitadas referentes à população adulta, sem distinção de idioma. Foram excluídos artigos publicados em formato de editorial.

Para a busca foram utilizados os descritores controlados em ciências da saúde (DeCS): Diagnóstico por Imagem; Segurança do paciente; Tomografia e os descritores controlados do vocabulário Medical Subject Heading (MeSH), na língua inglesa: Diagnostic Imaging; Patient safety; Tomography. O cruzamento desses descritores ocorreu por meio do operador booleano AND em todas as bases de dados, na ordem a cima citada, da seguinte maneira: Tomography AND Patient safety; Diagnostic Imaging AND Patient safety; Diagnostic Imaging AND tomography AND Patient safety.

Os cruzamentos permitiram a obtenção de um total de 14852 artigos, sendo 7620 na SCOPUS, 816 na Medline, 6045 na Web of Science, 371 na CINAHL. A seguir, será apresentada uma figura (1) com o panorama 
geral dos artigos selecionados.

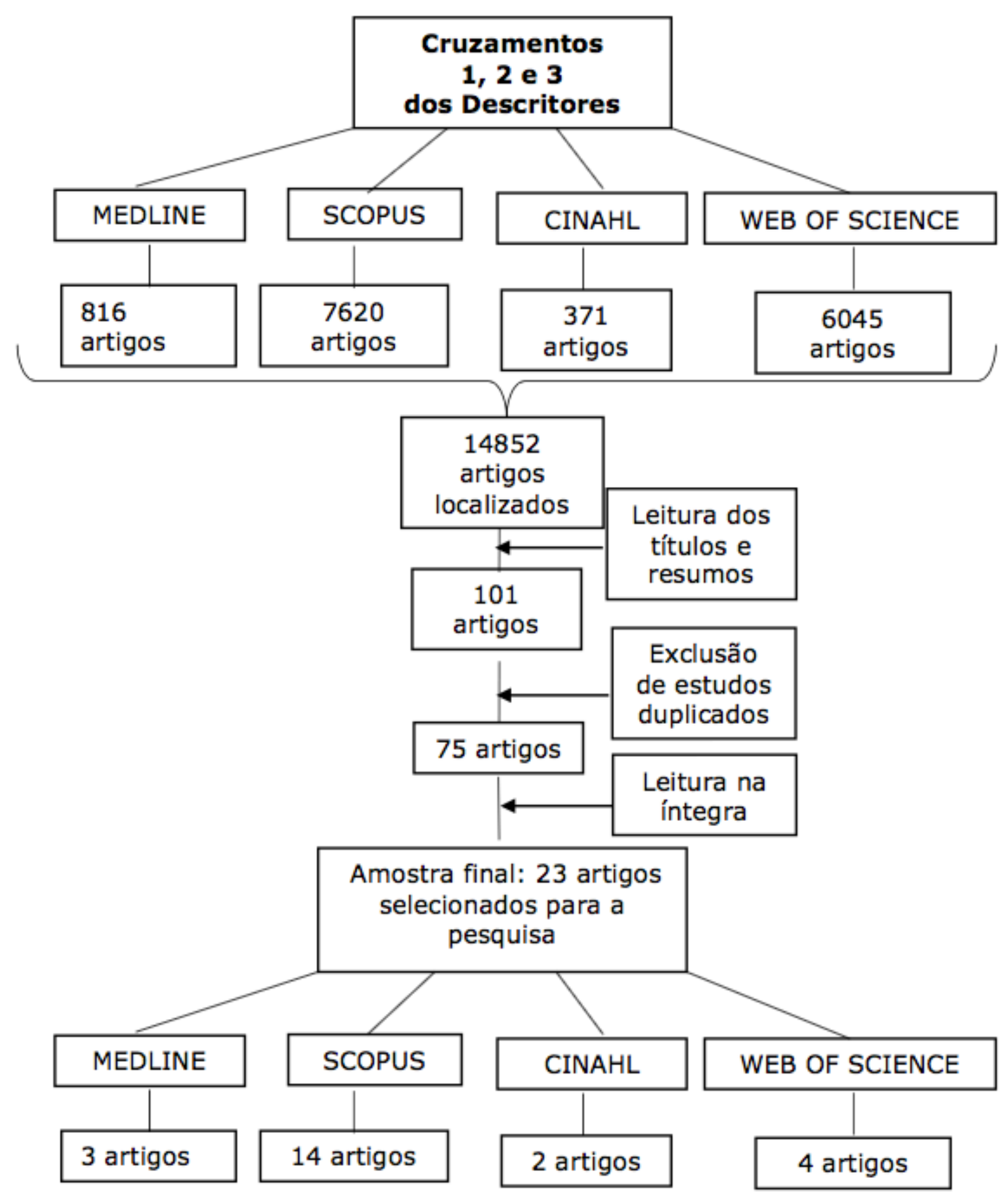

Figura 1: Diagrama de fluxo da seleção e inclusão dos artigos na revisão integrativa de literatura.

As publicações encontradas foram pré-selecionadas a partir da leitura dos títulos e resumos. Após a leitura na íntegra e análise dos artigos previamente selecionados, obedecendo aos critérios de inclusão, obteve-se uma amostra final de 23 artigos.

Para a extração de dados dos artigos incluídos na revisão integrativa, utilizou-se um instrumento 
validado, contendo a identificação do artigo; a introdução e objetivos; as características metodológicas; os resultados e as conclusões ${ }^{(15)}$.

O nível de evidência atribuído aos artigos baseou-se na seguinte classificação:

- Nível I- evidências resultantes de revisões sistemáticas ou meta-análise de ensaios clínicos;

- Nível II - evidências oriundas de no mínimo um ensaio clínico randomizado bem delineado;

- Nível III - ensaios clínicos sem randomização;

- Nível IV - estudos de coorte e caso-controle com um bom delineamento;

- Nível V-revisão sistemática de estudos descritivos e qualitativos;

- Nível VI - evidências provenientes de um único estudo descritivo e qualitativo;

- Nível VII - evidências baseadas em opinião de autoridades ou comitês de especialistas ${ }^{(16)}$.

Os estudos obtidos foram lidos na íntegra de forma a atender ao preenchimento do instrumento de coleta de dados. As informações colhidas passaram por um processo de dupla digitação na planilha do programa Microsoft Excel $2013^{\circledR}$ com o intuito de identificar inconsistências e minimizar o risco de erros, possibilitando uma melhor qualidade da revisão. Dessa forma, as informações contidas nos artigos selecionados, foram digitadas e redigitadas. Caso houvesse discordância de dados, realizava-se a correção procedendo a uma nova leitura do artigo que apresentasse discrepância de informações.

Por fim, procedeu-se a leitura exaustiva dos artigos com posterior categorização por conteúdo temático. Nesse processo, classificamos a informação textual, reduzindo-a em dados que eram relevantes, de modo a serem classificados e agrupados em categorias, que continham os mesmos significados e permitisse alcançar o objetivo proposto. Essa divisão foi meramente didática para a apresentação dos resultados e melhor entendimento da síntese do conhecimento desta revisão.

\section{RESULTADOS}

A organização, sumarização, análise crítica e integrativa das informações dos artigos sugeriu a sistematização dos artigos em três categorias que indicam os elementos essenciais para oferecer uma assistência segura aos pacientes submetidos ao exame de tomografia computadorizada, a partir de evidências da literatura: a otimização, monitoramento, qualidade e proteção em relação às doses de exposição à radiação; reações adversas e nefropatias relacionado ao uso do contraste; cuidados de enfermagem, gestão e casos específicos. A seguir, serão apresentados três quadros síntese que ilustram a análise dos artigos selecionados. 
Quadro 1: Síntese dos artigos da categoria Otimização, monitoramento, qualidade e proteção em relação às doses de exposição à radiação. Natal, RN, Brasil, 2015.

\begin{tabular}{|c|c|c|c|}
\hline Autor/periódico/ano/nível de evidência & Objetivo do estudo & Método da pesquisa & Síntese da evidência do artigo \\
\hline $\begin{array}{l}\text { Reiner BI, American College of } \\
\text { Radiology/2009/IV }\end{array}$ & $\begin{array}{l}\text { Quantificar os riscos da radiação, desenvolver padrões e diretrizes } \\
\text { baseadas nas "melhores práticas" de radiação e desenvolver novas } \\
\text { tecnologias para minimizar as doses de radiação. }\end{array}$ & Estudo de coorte. & $\begin{array}{c}\text { Manter a qualidade da imagem incluindo questões relativas } \\
\text { à segurança do paciente. }\end{array}$ \\
\hline $\begin{array}{l}\text { MacGregor K, Li I, Dowdell T, Gray BG/ } \\
\text { Radiology/2015/IV }\end{array}$ & $\begin{array}{c}\text { Avaliar esforços de protocolos de otimização de radiação para } \\
\text { tomografia computadorizada (TC) e determinar os níveis de dose } \\
\text { de radiação usando software de monitoramento de índice de dose } \\
\text { de radiação automatizado. }\end{array}$ & $\begin{array}{l}\text { Estudo retrospectivo } \\
\text { observacional. }\end{array}$ & Redução e rastreamento de dose de radiação. \\
\hline $\begin{array}{l}\text { Gervaisea A; Esperabe-Vignaub F.; Pernina } \\
\text { M et al./ Journal de Radiology/2011/IV }\end{array}$ & $\begin{array}{l}\text { Avaliar o conhecimento dos médicos que prescrevem exames de } \\
\text { TC sobre a protecção contra as radiações dos pacientes. }\end{array}$ & Estudo de coorte & $\begin{array}{l}\text { Aperfeiçoamento do conhecimento sobre proteção de } \\
\text { radiação. }\end{array}$ \\
\hline $\begin{array}{l}\text { Holmberg O; Malone J; Rehani M et al/ } \\
\text { European Journal of Radiology/ 2010/IV }\end{array}$ & $\begin{array}{l}\text { Associar a radiação ionizante com os riscos devido aos efeitos } \\
\text { indeterminados e considerar a protecção dos doentes de perigos } \\
\text { potenciais. }\end{array}$ & Estudos de coorte & Desenvolvimento de novas tecnologias visando a segurança. \\
\hline $\begin{array}{l}\text { Siegelman JRPW; Gress DA/ Journal of } \\
\text { American College Radiology/ 2013/IV }\end{array}$ & $\begin{array}{l}\text { Avaliar a eficácia de uma comissão multidisciplinar para estimar } \\
\text { dose e custos da realização de uma TC e descrever uma iniciativa } \\
\text { de melhoria da qualidade da radiação. }\end{array}$ & $\begin{array}{l}\text { Estudo de caso- } \\
\text { controle }\end{array}$ & $\begin{array}{l}\text { Protocolos de redução de dose de radiação do paciente, } \\
\text { melhoram a qualidade da assistência ao paciente, } \\
\text { desenvolvem uma cultura de segurança e diminuem custos. }\end{array}$ \\
\hline $\begin{array}{l}\text { Fletcher JG; Kofler JM; Coburn, JA et al./ } \\
\text { Abdominal Imaging Journaul/ 2013/V }\end{array}$ & $\begin{array}{l}\text { Refletir sobre a conscientização e comunicação sobre as questões } \\
\text { relacionadas com a dose de radiação. }\end{array}$ & $\begin{array}{l}\text { Revisão sistemática } \\
\text { de estudos } \\
\text { descritivos }\end{array}$ & $\begin{array}{l}\text { Individualizar tomografias de acordo com as características } \\
\text { do paciente e a indicação de diagnóstico sem comprometer } \\
\text { um diagnóstico preciso. }\end{array}$ \\
\hline $\begin{array}{l}\text { Tsivian M; Abern MR; Yoo JJ et al./ Journal } \\
\text { of Endourology/2013/IV }\end{array}$ & $\begin{array}{l}\text { Quantificar a dose eficaz associada a CT e determinar como fatores } \\
\text { dos pacientes afectam a exposição à radiação. }\end{array}$ & $\begin{array}{l}\text { Estudo retrospectivo } \\
\text { observacional. }\end{array}$ & $\begin{array}{l}\text { Estratégias de gestão devem ser desenvolvidas para reduzir } \\
\text { a exposição à radiação ionizante. }\end{array}$ \\
\hline $\begin{array}{l}\text { Talati RK; Dunkin J; Parikhb S; Moore WH/ } \\
\text { Journal of American College Radiology/ } \\
\text { 2013/V }\end{array}$ & $\begin{array}{l}\text { Descrever os componentes do núcleo de uma estratégia de } \\
\text { monitoramento da dose universal e detalhes das plataformas } \\
\text { comerciais disponíveis. }\end{array}$ & $\begin{array}{l}\text { Revisão sistemática } \\
\text { de estudos } \\
\text { descritivos }\end{array}$ & $\begin{array}{l}\text { Monitoramento, registro e comunicação de dose cumulativa } \\
\text { para os pacientes e profissionais resulta em maior } \\
\text { fidelidade do paciente, satisfação dos médicos e } \\
\text { oportunidade para novos negócios. }\end{array}$ \\
\hline $\begin{array}{l}\text { Raman SP; Mahesh M; Blasko, RV et al/ } \\
\text { Journal of American College Radiology/ } \\
\text { 2013/IV }\end{array}$ & Orientar radiologistas para reduzir a dose de radiação do paciente. & $\begin{array}{l}\text { Estudo de Caso } \\
\text { controle }\end{array}$ & $\begin{array}{c}\text { Entender melhor a tecnologia é essencial para a produção } \\
\text { de imagens de diagnóstico em doses mais baixas e uma } \\
\text { imagem segura. }\end{array}$ \\
\hline $\begin{array}{l}\text { Birnbaum, S./ Seminars Ultrasound CT } \\
\text { MRI/2010/V }\end{array}$ & $\begin{array}{c}\text { Minimizar a dose de radiação para pacientes que realizam } \\
\text { tomografia computadorizada. }\end{array}$ & $\begin{array}{l}\text { Revisão sistemática } \\
\text { de estudos } \\
\text { descritivos }\end{array}$ & $\begin{array}{l}\text { Os profissionais devem ter uma força política, social e } \\
\text { econômica para gerir redução de dose. }\end{array}$ \\
\hline $\begin{array}{l}\text { Qiao, Y.; Wang N.; Chen, R. et al / } \\
\text { American College of Radiology/ 2014/ IV }\end{array}$ & $\begin{array}{l}\text { Proporcionar novas evidências para o estabelecimento de uma } \\
\text { política de proteção radiológica para os pacientes. }\end{array}$ & Estudo coorte & $\begin{array}{l}\text { Compreensão do papel dos fatores de nível social em } \\
\text { proteção radiológica. lacuna entre a consciência e o } \\
\text { comportamento de segurança de radiação. }\end{array}$ \\
\hline
\end{tabular}

Rev. Eletr. Enf. [Internet]. 2016 [acesso em:_/___];18:e1189. Disponível em: http://dx.doi.org/10.5216/ree.v18.35312. 


\begin{tabular}{|c|c|c|c|}
\hline Autor/periódico/ano/nível de evidência & Objetivo do estudo & Método da pesquisa & Síntese da evidência do artigo \\
\hline $\begin{array}{l}\text { Yu JPJ, Kansagra AP; Mongan J/ American } \\
\text { College of Radiology/ 2014/IV }\end{array}$ & $\begin{array}{l}\text { Determinar a experiência dos radiologistas, analisando o tempo } \\
\text { absoluto e frequência eles passam no telefone para entender } \\
\text { melhor esse fenômeno e suas implicações potenciais. }\end{array}$ & $\begin{array}{l}\text { Estudo de Caso } \\
\text { controle }\end{array}$ & $\begin{array}{l}\text { Efeitos de interrupções freqüentes sobre o desempenho dos } \\
\text { radiologistas de plantão em instituições acadêmicas. }\end{array}$ \\
\hline
\end{tabular}

Quadro 2: Síntese dos artigos da categoria Reações adversas e nefropatias relacionadas ao contraste utilizado na tomografia computadorizada. Natal, RN, Brasil, 2015.

\begin{tabular}{|c|c|c|c|}
\hline Autor/periódico/ano/nível de evidência & Objetivo do estudo & $\begin{array}{l}\text { Método da } \\
\text { pesquisa }\end{array}$ & Síntese da evidência do artigo \\
\hline $\begin{array}{l}\text { Kobayashi D; Takahashi O; Ueda T et al / } \\
\text { BioMedCentral Medical Informatics and } \\
\text { Decision Making/ 2013/IV }\end{array}$ & $\begin{array}{l}\text { Criar uma regra de pontuação para prever reações } \\
\text { adversas aos meios de contraste utilizados na } \\
\text { tomografia computadorizada }\end{array}$ & Estudo de coorte & $\begin{array}{l}\text { Foram preditores significativos de uma reacção adversa aguda: reação } \\
\text { adversa prévia aos meios de contraste, urticária, história de alergia a outros } \\
\text { de agentes de contraste, agente de contraste de alta concentração, idade } \\
\text { maior que } 50 \text { anos e total de agente de contraste de doses maior que } 65 \mathrm{~g} \text {. }\end{array}$ \\
\hline $\begin{array}{l}\text { Piechowiak El; Peter JF; Kleb B; Klose KJ; } \\
\text { Heverhagen JT/ Radiology/2015/IV }\end{array}$ & $\begin{array}{c}\text { Determinar o efeito da utilização de agentes de } \\
\text { contraste iodados na formação de ADN de quebras } \\
\text { de cadeia dupla durante a tomografia de tórax } \\
\text { computadorizada (TC). }\end{array}$ & Estudo de coorte & $\begin{array}{c}\text { A aplicação de agentes de contraste iodados durante processos de raios-x de } \\
\text { diagnóstico, como tomografia computadorizada, conduz a um aumento do } \\
\text { nível de danos no DNA induzida por radiação. }\end{array}$ \\
\hline $\begin{array}{l}\text { Acauan LV; Rodrigues, MCS/ Texto } \\
\text { Contexto Enfermagem/ 2014/ VI }\end{array}$ & $\begin{array}{l}\text { Compreender a percepção da equipe de } \\
\text { enfermagem sobre segurança do idoso na } \\
\text { administração do meio de contraste iodado. }\end{array}$ & $\begin{array}{l}\text { Descritivo } \\
\text { qualitativo }\end{array}$ & $\begin{array}{l}\text { A equipe de enfermagem entende que a segurança do idoso na realização do } \\
\text { exame está associada à sua capacidade de avaliar riscos para ocorrência de } \\
\text { reações adversas relacionadas à administração do radiofármaco, em que se } \\
\text { entrelaçam processos, tecnologias e interações humanas, implicando } \\
\text { práticas seguras. }\end{array}$ \\
\hline $\begin{array}{l}\text { Kidoh, M; Nakaura, T; Awai K et al/ Journal } \\
\text { Computer Assisted Tomography /2014/II }\end{array}$ & $\begin{array}{c}\text { avaliar a segurança de um protocolo de baixa dose } \\
\text { para (TC) para pacientes com insuficiência renal por } \\
\text { nefropatia induzida por contraste. }\end{array}$ & $\begin{array}{l}\text { Ensaio clínico } \\
\text { randomizado }\end{array}$ & $\begin{array}{l}\text { A existência de protocolos de baixa dose de contraste permite realizar } \\
\text { tomografia com contraste, em pacientes com disfunção renal sem maiores } \\
\text { danos. }\end{array}$ \\
\hline $\begin{array}{l}\text { Velázquez MT, Albarrán A, Hernández F, } \\
\text { García Tejada J, Zueco J, Andreu J, et al/ } \\
\text { Acta Cardiologica/21010/II }\end{array}$ & $\begin{array}{l}\text { Analisar a eficácia diagnóstica e tolerabilidade do } \\
\text { uso iobitridol como agente de contraste } \\
\text { intravascular em diagnóstico por imagem, e revê de } \\
\text { forma breve as suas propriedades farmacológicas. }\end{array}$ & $\begin{array}{l}\text { Ensaio clinico } \\
\text { duplo cego } \\
\text { multicentrico }\end{array}$ & $\begin{array}{l}\text { lobitridol é um agente intravascular eficaz para o uso em diagnóstico por } \\
\text { imagem. }\end{array}$ \\
\hline $\begin{array}{l}\text { Traub, SJ; Mitchell, AM; Jones, AE et al/ } \\
\text { Annals of Emergency Medicine/ 2013/II }\end{array}$ & $\begin{array}{c}\text { Testar a hipótese de que a } \mathrm{N} \text {-acetilcisteína e solução } \\
\text { salina normal é mais eficaz do que a solução salina } \\
\text { normal só na prevenção de nefropatia por } \\
\text { contraste. }\end{array}$ & $\begin{array}{l}\text { Ensaio clinico } \\
\text { Duplo cego } \\
\text { randomizado }\end{array}$ & $\begin{array}{l}\text { Não foram encontradas evidência de um benefício para a administração N- } \\
\text { acetilcisteína aos pacientes submetidos a TC com contraste, no entanto } \\
\text { existe uma associação significativa entre o volume de fluidos intravenosos } \\
\text { administrados e redução na nefropatia induzida por contraste }\end{array}$ \\
\hline
\end{tabular}

Rev. Eletr. Enf. [Internet]. 2016 [acesso em:_____];18:e1189. Disponível em: http://dx.doi.org/10.5216/ree.v18.35312. 
Quadro 3: Síntese dos artigos da Categoria Cuidados de Enfermagem e gestão de casos especiais. Natal, RN, Brasil, 2015.

\begin{tabular}{|c|c|c|c|}
\hline Autor/periódico/ano/nível de evidência & Objetivo do estudo & Método da pesquisa & Síntese da evidência do artigo \\
\hline $\begin{array}{l}\text { Hussein AA; Abutaleb A; Jeudy, J et al./ Journal of } \\
\text { the American College of Cardiology/2014/IV }\end{array}$ & $\begin{array}{c}\text { Avaliar a segurança de tomografia computadorizada (TC) em } \\
\text { pacientes com equipamentos de gerenciamento do ritmo } \\
\text { cardíaco. }\end{array}$ & Estudo de coorte & $\begin{array}{l}\text { Não houve mudança na voltagem da bateria ou } \\
\text { parâmetros dos dispositivos de gerenciamento do ritmo } \\
\text { cardíaco expostos à radiação. }\end{array}$ \\
\hline $\begin{array}{l}\text { Litmanovich DE; Tack D; Lee KS et al/ Journaul } \\
\text { Thoracic Imaging/ 2014/V }\end{array}$ & \begin{tabular}{|c|} 
Rever as atuais recomendações baseadas em evidências para \\
temas de radiologia a pacientes grávidas e lactantes; fornecer \\
algoritmos práticos para minimizar o risco e aumentar a \\
segurança tanto para a gestante e para o feto.
\end{tabular} & $\begin{array}{l}\text { Revisão sistemática } \\
\text { de estudos } \\
\text { descritivos }\end{array}$ & $\begin{array}{l}\text { Para manter um alto nível de segurança em pacientes } \\
\text { grávidas, a radiação de imagem deve ser aplicada em } \\
\text { níveis tão baixos quanto possível. }\end{array}$ \\
\hline $\begin{array}{l}\text { Miguel C; Barros F S; Tilly JGJ; Fontoura LD; Sowek } \\
\text { LF; Saskoski GVR/ Colégio Brasileiro de Radiologia } \\
\text { e diagnóstico por imagem/ 2013/V }\end{array}$ & $\begin{array}{l}\text { Fornecer um subsídio sobre os elementos essenciais de } \\
\text { posicionamento do paciente para procedimentos de } \\
\text { radiologia. }\end{array}$ & $\begin{array}{l}\text { Revisão sistemática } \\
\text { de estudos } \\
\text { descritivos }\end{array}$ & $\begin{array}{l}\text { O posicionamento adequado do paciente deve ser } \\
\text { incluído na orientação dos enfermeiros que trabalham } \\
\text { com radiologia, porque a segurança do paciente é o } \\
\text { objetivo final em cuidar de todos os pacientes. }\end{array}$ \\
\hline $\begin{array}{l}\text { Campbell, KL; Hud, LM; Adams S et al/ The } \\
\text { American Journal of Medicine/ 2012/ IV }\end{array}$ & $\begin{array}{l}\text { Avaliar os resultados adversos após a administração de } \\
\text { contraste em uma coorte de pacientes com doença } \\
\text { falciforme. }\end{array}$ & Estudo de coorte & $\begin{array}{c}\text { Eventos adversos relacionados ao contraste intravenoso } \\
\text { ocorrerem em pacientes com doença falciforme, a uma } \\
\text { taxa semelhante à da população em geral. }\end{array}$ \\
\hline $\begin{array}{l}\text { Brask KB; Birkelund RP/ Jornaul Radiology } \\
\text { Nursing/ 2014/V }\end{array}$ & $\begin{array}{l}\text { Investigar como a equipe a experiência de cuidado expressa } \\
\text { durante o breve encontro com os pacientes em um } \\
\text { departamento de diagnóstico por imagem. }\end{array}$ & $\begin{array}{l}\text { Revisão sistemática } \\
\text { de estudos } \\
\text { descritivos e } \\
\text { qualitativo. }\end{array}$ & $\begin{array}{c}\text { O cuidado compreende as fases preparatórias e até que } \\
\text { a imagem seja encaminhada eletronicamente. }\end{array}$ \\
\hline Santosa AM; Martín JMA/ Radiología/ 2011/V & $\begin{array}{c}\text { Refletir sobre a demanda para a radiologia de emergência que } \\
\text { envolve mais de } 50 \% \text { da atividade global de serviços de } \\
\text { radiologia. }\end{array}$ & $\begin{array}{l}\text { Revisão sistemática } \\
\text { de estudos } \\
\text { descritivos }\end{array}$ & $\begin{array}{l}\text { Os hospitais devem ter uma unidade de radiologia de } \\
\text { emergência específica, equipada com meios para } \\
\text { cumprir essa missão. }\end{array}$ \\
\hline
\end{tabular}




\section{DISCUSSÃO}

A maior parte dos artigos selecionados foram localizados na SCOPUS, seguidos da Web of Science, PUBMED e CINAHL. Percebeu-se uma distribuição crescente das publicações entre os anos de 2013 e 2014, provavelmente por ser um assunto que vem sendo amplamente discutidos nos últimos anos.

Em relação ao idioma em que os estudos foram publicados, prevaleceu o inglês e em relação a categoria profissional, a médica. Esses profissionais estão envolvidos em todo o percurso do exame de TC, que vai desde a indicação/solicitação, sua execução, até a emissão de laudos. As evidências encontradas foram, na sua maioria, classificadas com nível quatro e cinco, entende-se que há uma necessidade de melhorar os estudos.

Os principais resultados foram organizados em três categorias, que serão descritas a seguir. A primeira tem a metade dos artigos inclusos: Otimização, monitoramento, qualidade e proteção em relação as doses de exposição à radiação ${ }^{(11-13 ; 17-19)}$. A outra metade dos estudos foram divididos igualmente nas seguintes categorias: Contraste utilizado na tomografia computadorizada, no que diz respeito a reações adversas e nefropatias $^{(20-25)}$ e Cuidados de enfermagem, gestão e casos específicos como a realização de exames em gestantes, pacientes com anemia falciforme e em uso de marcapassos ${ }^{(26-31)}$.

\section{Otimização, monitoramento, qualidade e proteção em relação as doses de exposição à radiação}

Os estudos analisados permitiram constatar que embora os grandes avanços da medicina, baseada no diagnóstico por imagens tenham permitido a identificação com maior acurácia e o tratamento de doenças em sua fase inicial, observa-se em contra partida, a possibilidade de erros com consequências danosas para a vida do paciente. Estas podem estar relacionadas a falhas na interpretação de exames ou excesso de confiança na imagem deixando-se de lado a clínica e a subjetividade do cliente. Estudos apontam que existe uma crescente evidência de maior risco de malignidade de órgãos sólidos e do sistema hemático com o aumento da exposição à radiação, mesmo em baixa doses, o que seria um aspecto negativo em relação ao rápido crescimento da utilização de tomografia computadorizada ${ }^{(17-19,32)}$

Neste contexto, também torna-se importante destacar que é comum a falta de segurança à radiação entre os profissionais que trabalham no ambiente hospitalar, seja por atos ou condições inseguras de trabalho. Os estudos existentes demonstraram uma falta de conhecimento sobre a dose, exposição e risco da radiação entre muitos profissionais da saúde ${ }^{(19,33)}$.

Com essa realidade, o governo dos Estados Unidos da América e de outros países, têm demonstrado uma vontade crescente de intervir nos cuidados de saúde nos últimos anos, particularmente quanto a segurança do paciente que recebe radiação durante a realização de exames como TC. Para tanto, fornecem normas regulamentadoras de segurança para a implementação de cuidados em saúde para os profissionais e instituições que trabalham com imagem ${ }^{(34)}$.

Com o uso dessas normas regulamentadoras, é possível implementar a estratégia de redução da dose de radiação, permitindo uma abordagem mais centrada no paciente. $O$ registro desses níveis de doses de 
radiação em pacientes, também permite que as instituições façam comparações detalhadas de seus níveis de dose de radiação, com intuito de determinar se eles estão mantendo a redução da dose na prática de acordo com as normas de segurança ${ }^{(35)}$.

A maioria dos protocolos requerem doses mais baixas em comparação com a técnica em uso na prática, para a obtenção da qualidade da imagem suficiente para permitir um diagnóstico com precisão ${ }^{(18,36)}$. Os fabricantes de tomógrafos, em resposta a questões de segurança, a crescente demanda pelo exame e preocupações médico-legais, desenvolveram múltiplas estratégias para diminuir a radiação por exame, tais como: protocolos padronizados, modulação automática de dose e algoritmos de reconstrução interativa. Radiologistas devem capacitar seus tecnólogos, técnicos de radiologia e biomédicos para se tornarem mais do que pressionadores de botões ${ }^{(37)}$.

Ainda neste contexto, com o aumento da atenção da mídia para erros de realização de exames e do aumento de freqüência de solicitação dos médicos desses exames para obter diagnósticos, tem-se observado um aumento na quantidade de publicações sugerindo estratégias de redução da dose de TC, as quais tem possibilitado na prática uma tendência de queda nas doses utilizadas. Porém, apesar desses esforços terem sido utilizados em alguns países, eles podem não se aplicar a todos os pacientes que necessitam de exames de TC devido as suas mais variadas condições clínicas ${ }^{(38)}$.

Desta forma, embora a redução de doses de radiação nos pacientes proporcionem contribuições valiosas para a melhoria da qualidade do exame, alguns cuidados devem ser tomados para evitar sua má utilização, pelos médicos, na tomada de decisões ao solicitar exames de imagem para os pacientes. Os profissionais de saúde devem ser cuidadosamente informados sobre o histórico desses usuários e ter domínio sobre a realização de procedimentos que utilizam tal tecnologia, antes da sua aplicação(35).

\section{Reações adversas e nefropatias relacionadas ao contraste utilizado na tomografia computadorizada}

Atualmente, uma nova filosofia organizacional fundamentada na segurança do paciente tem envolvido as instituições de saúde em todo o mundo. Estudos analisados onde a segurança do paciente é aspecto decisivo na assistência à saúde, tentam antecipar a ocorrência de erros antes que provoquem danos aos pacientes ${ }^{(20-25,31)}$.

Neste contexto, deve-se atentar para a incidência de reações adversas agudas aos agentes de contraste tilizados na TC, a qual é de aproximadamente 2 a $3 \%$, com contrastes de baixa osmolaridade. Os sintomas são diversos, variando de reações mais simples como rubor, prurido, uriticaria, e angioedema, até efeitos mais graves, reslacionados a hipotensão severa, perda de consciência, broncoespasmo e risco de vida por obstrução de vias aéreas ${ }^{(20)}$.

Uma pesquisa realizada na Alemanha, com 9.515 pacientes, demonstrou que as reações adversas medicamentosas foram relatadas por 70 pacientes $(0,74 \%)$, incluindo reações de hipersensibilidade em 55 deles. Apenas 30, apresentaram reações imediatas e 40 tiveram as tardias. Reações adversas sérias foram identificadas em cinco pacientes (0,05\%). Ainda nesse estudo, os pacientes com histórico de alérgias 
pareciam estar em maior risco de apresentar reações adversas imediatas e tardias. Porém, o desconforto foi geralmente leve, relatados por $72 \%$ dos pacientes ${ }^{(39)}$.

Além disso, em outra pesquisa, a reação adversa, como náuseas e vômitos, foi apenas observada em um de 108 pacientes $(0,93 \%)$ com o uso de contrastes de baixa osmolaridade, em comparação com um estudo prévio que estimava a ocorrência de $5,1 \%{ }^{(21)}$.

Dessa forma, conforme os artigos analisados, alguns fatores podem ser considerados como preditores de reações adversas, quais sejam: histórico de alergia a agentes de contraste, urticária, história de alergia anterior a outras drogas, concentração do agente de contraste maior do que $70 \%$, idade menor que 50 anos, dose total agente de contraste maior do que 65 g. A utilização dessas informações, podem facilitar encaminhamentos para uma melhor assistencia proporcionada pela equipe multiprofissinal, bem como o acompanhamento de pacientes de alto risco por médicos radiologistas ${ }^{(20)}$.

No que diz respeito à nefropatia induzida por contraste (NIC), é uma potencial complicação da administração deste, por meio da via intravenoso. Na população em geral, a incidência de NIC é estimada em 1 e $6 \%$. No entanto, o risco pode ser maior, chegando a $50 \%$ em alguns subgrupos de pacientes, como os portadores de miastenia gravis ${ }^{(40)}$.

Porém, alguns estudos demonstram que não há diferenças significativas na incidência de lesão aguda renal quando se utiliza uma dose baixa de contraste, uma vez que, nenhum dos pacientes com disfunção renal precisou realizar diálise após o procedimento. Dessa forma, um protocolo que utilize baixas doses de contraste pode permitir realizar o exame nesta população sem grandes preocupações com a sua segurança. Portanto, deve-se levar em consideração que, a freqüência de disfunção renal aguda pela NIC são raros ${ }^{(21)}$.

Quanto à utilização de contraste no exame de TC, outro fator importante de ser discutido é o protocolo de proteção renal. As investigações analisadas não demostraram evidências de benefícios para a administração $\mathrm{N}$-acetilcisteína aos pacientes submetidos a TC com contraste. No entanto, foi encontrada uma associação significativa entre o volume de fluidos intravenosos administrados e redução na nefropatia induzida por contraste ${ }^{(23)}$.

Cuidados de enfermagem e gestão de casos de exames em populações específicas como: gestantes, portadores de anemia falciforme, miastenia graves e marcapassos

A radiologia deixou de ser puramente uma especialidade de diagnóstico para medicina invasiva, processual, e curativa. Como resposta, o papel do enfermeiro em radiologia evoluiu. Hoje, o enfermeiro da radiologia é elemento integrante da assistência aos pacientes submetidos a procedimentos radiológicos ${ }^{(22)}$.

Neste sentido é fundamental que o enfermeiro tenha habilidades técnicas como: posicionamento seguro do paciente, habilidades de cuidados críticos, pensamento crítico e habilidades de avaliação clínica, manuseio de materiais e equipamentos, habilidades em situações de urgência e emergência e de cuidados ambulatoriais. Também são necessários, recursos humanos adequados, educação permanente e indicadores de qualidade confiáveis para avaliação da assistência ${ }^{(22,28,41)}$. 
Neste contexto, a Food and Drug Administration (FDA) vem exigindo uma melhor gestão e organização, onde as informações para o usuário sejam mais abrangentes, com um foco particular na garantia de qualidade. Institui ainda que as instalações de exames por imagem devem seguir as normas regulamentadoras para evitar eventos adversos e os riscos evitáveis ${ }^{(30-31)}$. Destacou também três áreas de foco em segurança na tomografia computadorizada: erro médico, utilização correta de TC e otimização de $\operatorname{dose}^{(33)}$.

No que diz respeito aos exames de imagens em pacientes grávidas, exige familiaridade com tópicos importantes, tais como: risco de exposição à radiação para a mãe, o risco de exposição à radiação indireta para o feto, dosimetria fetal e materna. A segurança na administração de contraste iodado durante a gravidez e lactação também são essenciais. É fundamental entender que durante a gravidez dois indivíduos são expostos simultaneamente, e que a intensidade das consequências de exposição podem variar substancialmente entre os dois. O Colégio Americano de Radiologia e Congresso Americano de Obstetras e Ginecologistas concordam que o exame de imagem deve ser realizado depois do julgamento clínico de risco/benefício e o nível de radiação deve ser mantido tão baixa quanto razoavelmente realizável ${ }^{(27)}$.

No que se refere a pacientes com doença falciforme, os eventos adversos relacionados com contraste intravenoso podem ocorrer em uma taxa semelhante ao da população em geral, sem aumento de NIC. Portanto, o uso do diagnóstico por imagem pode ser realizado sem aumento de risco de complicação grave nesta população ${ }^{(29)}$.

Quanto aos pacientes portadores de gerenciamento do ritmo cardíaco, muitos foram submetidos a esse exame, não sendo constatados casos na prática, de eventos adversos, nem descritos para as autoridades ou publicados em artigos, tornado a realização de TC segura para pacientes que portam estes dispositivos ${ }^{(26,42)}$.

Outro estudo demostrou que não há aumento imediato do risco de exacerbação da fraqueza miastênica com o uso de contraste de baixa osmolaridade, o que se opõe quando comparado com estudos anteriores que utilizaram meios de contraste iónicos e mostraram aumento da fraqueza entre 2,1 e 3,4\% de pacientes. Logo, embora este estudo sugira que não há aumento do risco imediato de agravamento dos sintomas miastênicos nessa população, devem ser tomadas precauções na administração de meios de contraste em pacientes com Miastenia Gravis ${ }^{(40)}$.

\section{CONCLUSÃO}

Este estudo permitiu sintetizar achados relacionados aos elementos essenciais para oferecer uma assistência segura aos pacientes submetidos à tomografia computadorizada, cujas evidências foram distribuídas em categorias, demonstrando seu impacto para a prática clínica.

A categoria "otimização, monitoramento, qualidade e proteção em relação às doses de exposição à radiação", demonstrou que as evidências para essa assistência partem da necessidade de minimizar a indicação de exames e exposição desnecessária à radiação, garantia da proteção radiológica para pacientes, 
profissionais e ambiente, bem como o desenvolvimento de novas tecnologias de segurança, sem interferir na qualidade de imagem.

No que diz respeito à categoria "reações adversas e nefropatias relacionadas ao contraste utilizado na tomografia computadorizada", evidenciou a necessidade do uso de protocolos com realização de exames de TC com baixas doses de radiação, em contra partida, identificou-se lacunas relacionadas à carência de estudos que explorem os preditores significativos de avaliação de riscos para ocorrência de uma reação adversa aguda, relacionadas à administração e concentração do contraste.

Por sua vez, a categoria "cuidados de enfermagem e gestão de casos de exames em populações específicas como: gestantes, portadores de anemia falciforme, miastenia graves e marcapassos", evidenciou a necessidade de mudanças nos serviços de tomografia, por meio da implementação de indicadores para avaliar qualidade da assistência e o desenvolvimento de iniciativas educacionais que estabeleçam programas que não só organizem o serviço, mas também qualifiquem profissionais por meio de processos de educação permanente na área da radiologia.

Por fim, ao identificar os elementos essenciais para oferecer uma assistência segura aos pacientes submetidos ao exame de tomografia computadorizada, baseadas nas melhores evidências científicas, o estudo poderá contribuir como subsidio teórico para a formulação de protocolos assistenciais para pacientes atendidos em serviços de tomografia computadorizada.

\section{REFERÊNCIAS}

1. Parente DB. Radiation risk in the indiscriminate use of CT scan. Radiol Bras. 2013;46(2):5-6.

2. Gabriel CS, Melo MRAC, Rocha FLR, Bernardes A, Miguelaci T, Silva MLP. Use of performance indicators in the nursing service of a public hospital. Rev. Latino-Am. Enfermagem. 2011;19(5):1247-54.

3.Sales OP, Oliveira CCC, Spirandelli MFAP, Cândido MT. Atuação de enfermeiros em um Centro de Diagnóstico por Imagem. J Health Sci Inst. 2010;28(4):325-8.

4.Navarro MVT, Costa EA, Drexler GG. Controle de riscos em radiodiagnóstico: uma abordagem de vigilância sanitária. Revista Ciência \& Saúde Coletiva. 2010;15(3):3477-86.

5. Reiner BI. Quantifying Radiation Safety and Quality in Medical Imaging. Creating the Infrastructure. American College of Radiology.2009;6(8):558-61.

6. MacGregor K, Li I, Dowdell T, Gray BG. Identifying Institutional Diagnostic Reference Levels for CT with Radiation Dose Index Monitoring Software. Radiology. 2015; 276(2): 507-17.

7. Gervaisea A, Esperabe-Vignaub F, Pernina M, Naulet P, Portron Y, Lapierre-Combes M. Evaluation of the knowledge of physicians prescribing CT examinations on the radiation protection of patients. Jornal de radiologie.2011;92(7-

8):681-7.

8. Holmberg O, Malone J, Rehani M, McLean D, Czarwinski R. Current issues and actions in radiation protection of patients. European Journal of Radiology. 2010;76:15-9.

9. Siegelman JRPW, Gress DA. Radiology Stewardship and Quality Improvement: The Process and Costs of Implementing a CT Radiation Dose Optimization Committee in a Medium-Sized Community Hospital System. J Am Coll Radiol.2013;10(6):416-22.

10. Fletcher JG, Kofler JM, Coburn JA, Bruining DH, McCollough $\mathrm{CH}$. Perspective on radiation risk in $\mathrm{CT}$ imaging. Abdom Imaging. 2013, 38:22-31.

11. Tsivian M, Abern MR, Yoo JJ, Evans P, Peter Qi, Kim CY, et al. Radiation Exposure Associated with Dedicated Renal Mass Computed Tomography Protocol: Impact of Patient Characteristics. Journal of Endourology. 2013; 27(9): 1102-6. 
12.Talati RK, Dunkin J, Parikhb S, Moore WH. Current Methods of Monitoring

Radiation Exposure From CT. J Am Coll Radiol. 2013; 10(9):702-7.

13. Raman SP, Mahesh M, Blasko RV, Fishman EK. CT Scan Parameters and Radiation Dose: Practical Advice for Radiologists. J Am Coll Radiol 2013;10:840-6.

14. Whittemore R. Combining evidence in nursing research: methods and implications. Nurs Res. 2005; 54(1):56-62. 15.Ursi, ES; Gavão, CM. Prevenção de lesões de pele no perioperatório: revisão integrativa da literatura. Revista Latino-Americana de Enfermagem. 2006; 14(1): 124-31.

16. Melnyk BM, Fineout-Overholt E, Stillwell SB, Williamson KM. Evidence-based practice: step by step: The Seven Steps of Evidence-Based Practice. Am j nurs. 2010; 110(1):51-3.

17. Birnbaum S. Radiation Protection in the Era of Helical CT: Practical Patient Based Programs for decreasing Patient Exposure. Semin Ultrasound CT MRI. 2010;31:46-52.

18. Qiao Y, Wang N, Chen R, Liang Y. Societal and Individual Determinants of Patients Radiation Safety Behavior: A Preliminary Investigation from China. American College of Radiology.2014;11(5):521-6.

19. Yu JPJ, Kansagra AP, Mongan J. The Radiologist's Workflow Environment: Evaluation of Disruptors and Potential Implications. American College of Radiology. 2014;11(6):589-593.

20. Kobayashi D, Takahashi O, Ueda T, Deshpande GA, Arioka H, Fukui T et al. Risk factors for adverse reactions from contrast agents for computed tomography. BMC Medical Informatics and Decision Making. 2013; 2013; 13(18):1-6.

21. Piechowiak El; Peter JF; Kleb B; Klose KJ; Heverhagen JT. Intravenous lodinated Contrast Agents Amplify DNA Radiation Damage at CT. Radiology. 2015; 275(3): 692-7.

22. Acauan LV, Rodrigues MCS. Percepções da equipe de enfermagem sobre segurança Do idoso na tomografia computadorizada cardíaca Contrastada. Texto Contexto Enferm. 2014; 23(2):338-46.

23. Kidoh M, Nakaura T, Awai K, Matsunaga Y, Tanoue K, Harada K, et al. Low-Contrast Dose Protection Protocol for Diagnostic Computed Tomography in Patients at High-Risk for Contrast-Induced Nephropathy. J Comput Assist Tomogr. 2013; 37(2):289-96.

24. Velázquez MT, Albarrán A, Hernández F, García Tejada J, Zueco J, Andreu J, et al. Less iodine injected for the same diagnostic performances: comparison of two low-osmolar contrast agents (iobitridol 350 and iopamidol 370) in coronary angiography and ventriculography: a randomized double-blind clinical study. Acta Cardiol. 2010;65(4):387-

94.

25. Traub SJ, Mitchell AM, Jones AE, Tang A, O'Connor J, Nelson T, et al. Intravenous Fluids Alone to Prevent ContrastInduced Nephropathy in Emergency Computed Tomography. Annals of Emergency Medicine. 2013. 62(5):511-520.

26. Hussein AA, Abutaleb A, Jeudy J, Phelan T, Patel R, Shkullaku M, et al. Safety of Computed Tomography in Patients With Cardiac Rhythm Management Devices. Journal of the American College of Cardiology, 2014;63 (17).

27. Litmanovich DE, Tack D, Lee KS, Shahrzad M, Bankier AA.Cardiothoracic Imaging in the Pregnant Patient. J Thorac Imaging. 2014; 29(1): 38-49.

28. Miguel C; Barros F S; Tilly JGJ; Fontoura LD; Sowek LF; Saskoski GVR. Análise de filmes radiográficos rejeitados em um hospital de grande porte. Colégio Brasileiro de Radiologia e diagnóstico por imagem. 2013. 46 (1): 129-137.

29. Campbell KL, Hud LM, Adams S, Andrel J, Ballas SK, Feldman AM, et al. Safety of lodinated Intravenous Contrast Medium Administration in Sickle Cell Disease. The American Journal of Medicine, 2012;125(1):100e11-6.

30. Brask KB, Birkelund RP. "Patient Care in Radiology"- The Staff's Perspective. J Radiol Nurs. 2014;33:23-9.

31. Santosa AM, Martín JMA. Organización y gestión de la radiología urgente. Radiología. 2011;53(1):7-15

32. Weinstein MS. Tackling the computed tomography epidemic. Journal of surgical research. 2013;84(1):796-7.

33. Saeed SA, Masroor I, Shafqat G. Learning from errors in radiology to improve patient safety. Journal of the College of Physicians and Surgeons Pakistan. 2013;23(10):691-4.

34. Harvey HB, Pandharipande JDP. The Federal Government's Oversight of CT Safety: Regulatory Possibilities. Radiology.2012;262(2):391-8.

35. Eisenberg JD, Harvey HB, Moore DA, Gazelle GS, Pandharipande PV. Falling Prey to the Sunk Cost Bias: A Potential Harm of Patient Radiation Dose Histories. Radiology. 2012; 263(3):626-8.

36. Hevezi JM, Mahesh M. Optimizing CT Dose and Image Quality for Radiotherapy Patients. American College of Radiology 2012; 11(1):152.

37. Birnbaum S. CT Dose Optimization: The General Radiologist's Perspective. American College of Radiology. 2013;10(8):637-638.

38. Mahesh M, Fishman EK. CT Dose Reduction Strategy: To Modulate Dose or Not in Certain Patients? American

Rev. Eletr. Enf. [Internet]. 2016 [acesso em:___/_];18:e1189. Disponível em: http://dx.doi.org/10.5216/ree.v18.35312. 
College of Radiology. 2012; 9(12):931-2.

39. Häussler M D. Safety and patient comfort with iodixanol: A postmarketing surveillance study in 9515 patients undergoing diagnostic CT examinations. Acta Radiologica. 2010;51(8):924-33

40. Mehrizi M, Pascuzzi R. Complications of radiologic contrast in patients with myasthenia gravis. Muscle \& nerve. 2014;50(3):443-4.

41. Sousa MF. Management and Leadership: Educating and Orienting the Radiology Nurse of the Future. Journal of Radiology Nursing. 2011;30(3):135-6.

42. Arbelo E, Brugada J. Cardiac Rhythm Management Devices. When Regulatory Agencies "Over-Regulate". Journal of the American College of Cardiology 2014; 63(17): 1776-7. 